Article

\title{
Optimal Volume for Concert Halls Based on Ando's Subjective Preference and Barron Revised Theories
}

Salvador Cerdá $^{1, *}$, Jaume Segura ${ }^{2}$, Alicia Giménez ${ }^{3}$, Arturo Barba ${ }^{3}$ and Rosa Cibrián ${ }^{4}$

1 Department of Experimental and Social Sciences Teaching, University of Valencia, Av. dels Tarongers s/n, Valencia 46022, Spain

2 Department of Computer Science, University of Valencia, Poligon de la Coma s/n, Paterna 46980, Spain; E-Mail: jaume.segura@uv.es

3 Department of Applied Physics, Polytechnic University of Valencia, Camino de Vera s/n, Valencia 46022, Spain; E-Mails: agimenez@fis.upv.es (A.G.); arbarse@hotmail.com (A.B.)

4 Department of Applied Physics, University of Valencia, Blasco Ibañez, Valencia 46010, Spain; E-Mail: rosa.m.cibrian@uv.es

* Author to whom correspondence should be addressed; E-Mail: cerdaj@uv.es; Tel.: +34-9638-77000; Fax: +34-9638-77179.

Received: 21 January 2014; in revised form: 13 March 2014 / Accepted: 18 March 2014 / Published: 27 March 2014

\begin{abstract}
The Ando-Beranek's model, a linear version of Ando's subjective preference theory, obtained by the authors in a recent work, was combined with Barron revised theory. An optimal volume region for each reverberation time was obtained for classical music in symphony orchestra concert halls. The obtained relation was tested with good agreement with the top rated halls reported by Beranek and other halls with reported anomalies.
\end{abstract}

Keywords: room acoustics; sound quality; optimal criterium

\section{Introduction}

Obtaining a criterion based on objective measures to assess the quality of a concert hall is one of the fundamental aims of room acoustics. However, recently, it has been recognized that one of the most important missing elements in the room acoustics puzzle is the lack of preferred design criteria for each room acoustics parameter [1]. Since Clemens Wallace Sabine [2], whose initial discovery of the 
reverberation time as a persistence of the sound in an enclosure constituted the most important parameter in the evaluation of a hall, new descriptors were added in the following decades to analyze and to evaluate existent halls or to design those in the building stage. A large list and chronological order of all parameters have been developed over the years; the original references were presented in [3].

There is no unique and definitive design parameter that can be said to correspond with perceived hall acoustic quality. However, different authors have tried to find models that combine the studied acoustic parameters with the aim of obtaining an objective ranking that correlates with the subjective ranking.

Otherwise, it is very important for designers to have a model that enables the prediction of subjective response from the objective measurements.

One of the most important references for determining optimal values from psycho-acoustic studies can be found in the works by Ando [4,5] on the preferred acoustic parameters of different musical criteria, and especially, his theory of subjective preference. He used as independent statistical parameters (variations in any of which do not affect the others): the interaural cross-correlation index (IACC), early decay time (EDT), strength factor (G), and the time between the first direct sound and the first reflection (ITDG). Using subjective preferences for the four orthogonal factors of the sound field that are common to most listeners, one can add individual factor preferences together to derive an overall estimated preference value for each seat [6]. Thus, a design procedure can be easily obtained.

Beranek added two independent parameters to those of Ando: bass ratio (BR) and the surface diffusion index (SDI). These parameters were used with a modification of Ando's theory to obtain an objective hall ranking method [7].

On the other hand, room acoustics is a well-established branch of acoustical engineering having its own standard. The ISO standard proposes that the acoustics of a hall can be described with just a few numbers obtained by spatially averaging over several measured seats. In the current decade, that standard has been criticized on many stages: the algorithms to compute the parameters are imprecise, the applied frequency range is too narrow, and a single omnidirectional source is a poor representation of the dozens of sound sources present in a real orchestra [1,8]. Moreover, there are many works that have shown measured parameters fail to describe the details of perceived acoustics [9-12].

In previous studies $[13,14]$ we reduced statistically, by factor analysis, the number of acoustic parameters to an orthogonal set of three factors. One factor was interpreted as RT, another as a spatiality factor correlated with lateral energy fraction (LFC); and the third was an envelopment factor correlated with listener envelopment (LEV according to $[15,16]$ ).

In [17], the authors presented a linear combination of RT, LFC and LEV correlating with Ando functions of preferred values. This allowed us to introduce an Ando-Beranek function fitted to the experimental objective data that agrees with the assessment of the halls included in our studies $[18,19]$. An optimal interval of the Ando-Beranek function was established too. This optimal interval involves $\mathrm{RT}_{\text {mid }}, \mathrm{LFC}_{\mathrm{E} 4}, G_{\text {late }}$ and $\mathrm{IACC}_{\mathrm{L} 3}$ (the last two parameters are used in LEV computation).

As shown in extensive objective acoustic measurements by Barron and Lee [20] in various concert halls in Britain, the total reflected sound energy does significantly fall off with increasing source-receiver-distance. This is due to the fact that listeners closer to the source not only receive a higher level of direct sound but also higher levels of early reflections because they have travelled shorter distances. In their paper of 1988 Barron and Lee, therefore proposed their revised theory of sound decay in concert spaces, which took account for these facts. This theory includes the Barron model for $G_{\text {late: }}$ : 


$$
G_{\text {late }}=10 \cdot \log \left(\frac{31200 \cdot R T}{V}\right)-\frac{4.82+0.174 \cdot r}{R T}
$$

Traditional statistics is strong in devising ways of describing data and inferring distributional parameters from sample. But, the obtained statistical models can be spurious or have a poor validity. A way to show validity of the model is to apply it to another data set. Albeit our model was computed using mean values of room parameters, in a recent work, we used Ando-Beranek's model to evaluate quality in a hall by using quality maps $[21,22]$, i.e., using the model at each measured point in the hall. The results were very interesting because the maps show three distinct areas: an area that satisfies the optimum criteria, and two others that provide either lower or higher criterion values. In the case that the values are lower (and the RT of the room is suitable for classical music), it is interpreted that there is a deficiency of late reflections. While in the opposite case, there is too late lateral energy.

In this work, we combine the results obtained in [17] for symphony orchestra concert halls, with the Barron's model. We obtain a formula for an optimum volume region according to the Ando-Beranek's model for each reverberation time. To test the validity of the obtained expression, we applied it to concert halls ranked in Beranek's work [23], halls in Barron and Lee work [20] and halls with bad sound quality reported [24].

\section{The Ando-Beranek's Model}

In this section we introduce the Ando-Beranek's model inspired by the work of these two authors [5,7]. This model consists of a linear combination of orthogonal parameters that correlate with a linear combination of $\mathrm{Si}$ Ando functions [4]. We chose a linear model because its simplicity, but other solutions could exist, like deviations from optimal values, non-linear (like quadratic or square root relationships, etc.) These possibilities will be considered in future works.

In Beranek [7], it is said that from Ando's studies one can deduce the relative weighting evolved for each $S_{\mathrm{t}}$ Ando's functions. We have introduced [17] weights constants as additional degrees of freedom. These degrees of freedom enable a linear combination $S_{\mathrm{AB}}$ of the orthogonal factors obtained in previous works $[13,14]$ that correlates with a linear weighted combination $S_{\mathrm{a}}$ of $S_{\mathrm{t}}$ functions $\left(S_{\mathrm{AB}}\right.$ was named Ando-Beranek's model).

The general problem to be solved is the following: how to find the coefficients in:

$$
S_{\alpha}=\sum_{i=1}^{5} \alpha_{i} \cdot S_{i}
$$

with

$$
\sum_{i=1}^{5} \alpha_{i}=1
$$

in such a way that the correlation coefficient:

$$
r=\operatorname{corr}\left(C+x R T_{\text {mid }}+y L F C_{\mathrm{E} 4}+z L E V, S_{\mathrm{a}}\right)
$$

is maximum. As the functions of Ando's functions are related to the subjective response for large concert halls and classical music, we expect that the linear combination obtained will provide equivalent 
information. This fact is not guaranteed from a mathematical point of view, so an experimental test is necessary to confirm it.

In [17], it was found that the combination with the best correlation was:

$$
S_{\mathrm{AB}}=-1.49+0.36 R T_{\text {mid }}+2.76 L F C_{\mathrm{E} 4}-0.19 \cdot L E V
$$

With regard to our subjective hall assessment [18], the hall quality criterion with the $S_{\mathrm{AB}}$ parameter was:

$$
-1 / 3<S_{\mathrm{AB}}<0
$$

This model and the quality criterion for symphony orchestra concert halls, supported by our experimental data, was used to mapping quality in a hall with satisfactory results $[21,22]$.

\section{The Optimal Volume Region for Halls: Deduction and Discussion}

At this point we must review the knowledge about volume in design room acoustic $[6,7,25,26]$. There are at least six ingredients that must be addressed if one is to provide good acoustics in a hall: Reverberance, Loudness, Clarity, Spatial Impression, Warmth and Intimacy. It is generally accepted that none of these on its own can guarantee good acoustics. Rather, each ingredient must be present and in its right proportion [1,27], like in a good meal.

The first step in acoustic design is to choose a reverberation time adequate to hall's use. After considering the volume and area following Sabine's theory, the next level of detail includes specific reflections. Certain 1st-order reflectors arrive at the listener from above. A low ceiling tends to promote low reverberance, lack of envelopment and a generally inadequate increase of loudness (as it directs sound into the absorbing audience). So, an important factor becomes Height/Width ratio or equivalently EDT/RT ratio [28]. An architectural solution may be found in adjusting the effective room cross-section to produce precedence for the lateral reflections [29].

In the early 60s, Leo Beranek postulated the importance of Initial Time Delay Gap, and this often led to arrays of small reflectors suspended below the ceiling. These allowed the ceiling to be higher, in order to sustain reverberance, but the reverberation was still addressed as a simply function of volume and area, or the number of seats.

Designs based on this approach resulted in several wide fan-shaped and oval halls with overhead reflectors. Some halls designed using these rules showed problems. The analysis of what these halls are missing is related to the loudness of the lateral sound, both early and late lateral sound. The importance of lateral reflections was advanced by Barron and Marshall. Note here that these arguments can be used as a support of the quality criterion 6 because our expression involves RT, LFC and LEV.

These properties depend on constructional data [25], in particular on the:

a shape of the room;

b volume of the room;

c number of seats and their arrangement;

d materials of walls, ceiling, floor, seats, etc.

While the reverberation time is determined by factors (b), (d) and not significantly by (a), the room shape influences strongly the number, directions, delays and strengths of the early reflections received at a given position or seat. The strength of the direct sound depends on the distances to be covered, and also on 
the arrangement of the audience. In specific reference to the volume, large room volume is necessary to get an adequate reverberance and a good spaciousness [26].

Beranek's design procedure use strength $G$ to determine audience area and, combined with reverberation, establish the hall's volume. In his procedure, it may go unnoticed reverb efficiency related to Height/Width ratio and the assumption that about $75 \%$ of the total sound absorbtion in a hall is contributed by the audience and orchestra [28]. Although Beranek did not combine their results into a single graph, it is easy to get the appropriate volume region using his criterion on the strength parameter. That is, $G_{\text {mid }} \in[3.5,5]$ dB. Figure 1 shows optimal region in Beranek's procedure and rooms in Beranek's book [23].

Figure 1. V-RT region following Beranek's design procedure. Concert Halls from his 2006 book are shown [23].

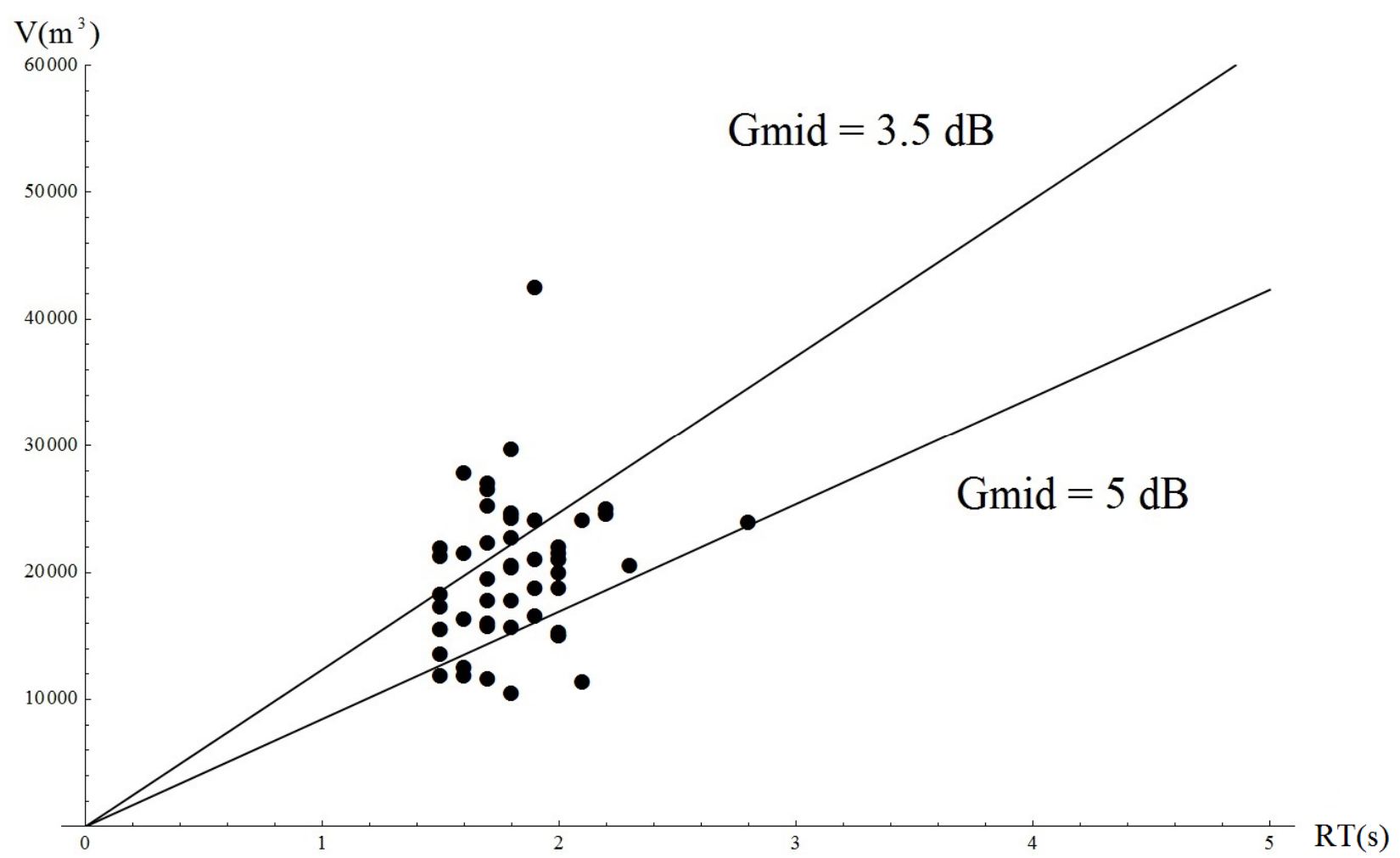

To evaluate the model of Ando-Beranek's and its optimum interval from the point of view of the acoustical design of concert halls for symphony orchestra music halls, we are going to consider some basic approximations from room acoustics. First of all, we consider the classical expression of the Sabine's reverberation time:

$$
R T=0.161 \cdot \frac{V}{A}
$$

In the quality relationship (6), some data from the room RT, LFC, IACCL, are considered design parameters. From the quality criterion we find that $G_{\text {late }}$ satisfy the inequalities:

$$
-\frac{694}{57}+\frac{72}{19} \cdot R T+\frac{552}{19} \cdot L F C-20 \log (1-I A C C L) \geq G_{\text {late }}
$$




$$
G_{\text {late }} \geq-\frac{298}{19}+\frac{72}{19} \cdot R T+\frac{552}{19} \cdot L F C-20 \log (1-I A C C L)
$$

We can use now the Barron's model [20]. In this model, it is considered that the energy can be calculated as direct, early and late sound, and thus, the value of the late acoustic strength $\left(G_{\text {late }}\right)$ will be given by the Equation (1). From the quality Equation (6), we can get an interval of variation for $G_{\text {late }}$ that we consider as optimum $\left[G_{\text {late;min, }}, G_{\text {late;max }}\right][13,14]$. This interval can be combined with the Barron's equation for the late energy to obtain an estimation of the volume. We just need to introduce a new parameter, which is the minimum distance from the seats to the source $r_{\min }$. This distance is where the $G_{\text {late }}$ will be maximum and, therefore, it will allow us to deduce the corresponding volume through the formula:

$$
V_{o p t, \max }=\frac{31200 \cdot R T}{10 \frac{G_{\text {late, } \max }+\frac{4.82}{R T}+\frac{0.174 \cdot r_{\min }}{R T}}{10}}
$$

According to the Barron's model, the variation of $G_{\text {late }}$ with distance is very slow $(-0.174 / T \mathrm{~dB} / \mathrm{m})$. This implies that, in case the condition for the minimum distance would be accomplished, it would be also fulfilled for the maximum distance of the hall. The range $\left[G_{\text {late;min, }}, G_{\text {late;max }}\right]$ is always $3.51 \mathrm{~dB}$ wide. This supposes that in case the minimum distance condition is fulfilled, the maximum accepted distance is:

$$
d_{\text {max }} \approx 20 \cdot T
$$

As a numerical example, we now consider as design values the following ones: $\mathrm{RT}=2.5 \mathrm{~s}, \mathrm{LFC}=0.2$ and IACCL $=0.15$. These values give an optimum interval for the late strength: $G_{\text {late }}=[1.01,4.52]$. If we consider a minimum distance of $8 \mathrm{~m}$, we found that the room volume is $15,544 \mathrm{~m}^{3}$. Furthermore, we have guaranteed the optimum interval of late energy up to a $50 \mathrm{~m}$ distance to the source, which is clearly enough.

Taking as design parameters LFC, IACCL and $r_{\text {min }}$, we can represent the Equation (3) for different values of reverberation time $\mathrm{T}$. This representation allows us to validate the obtained result by applying it to well-known halls.

Figure 2 shows optimal V-RT region for some design parameters (IACCL3 $=0.15, r_{\min }=7 \mathrm{~m}$ ). In a first sight, we can appreciate that the combination of the Barron's model with the quality Equation (6), gives an expression which satisfies the following limits:

$$
\lim _{R T \rightarrow 0} V(R T)=\lim _{R T \rightarrow+\text { inf }} V(R T)=0
$$

This means that out of the fitted interval of reverberation time, it is not meaningful to design rooms for classical music according to this result, because it would have a very little volume.

There are three groups of halls in Figure 2. We have included the first 10 best valued halls in Beranek's book [7]. For these halls, Figure 2 shows a good accord between the theoretical optimal region and their RT and Volume values. This is a remarkable result, because our relationship has been obtained from other rooms and with other methodology. We have also included the rooms used by Barron and Lee [20]. We can see that in their work there are small halls which are not in the optimum region shown in Figure 2. So we can argue that the goodness of the region is not derived exclusively from the 


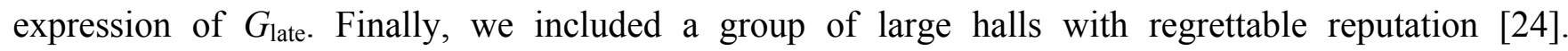
The acoustic of these halls are analyzed with detail in [24]. A general problem found is that the unfortunate combination of a large volume, a short Reverberation Time and long distances produce low G levels, Early Lateral Fractions are generally low and the Late Energy $\left(G_{\text {late }}\right)$ is low. As we can see in Figure 2, these halls are out of the obtained optimal area.

Figure 2. V-RT region following Ando-Beranek's formula.

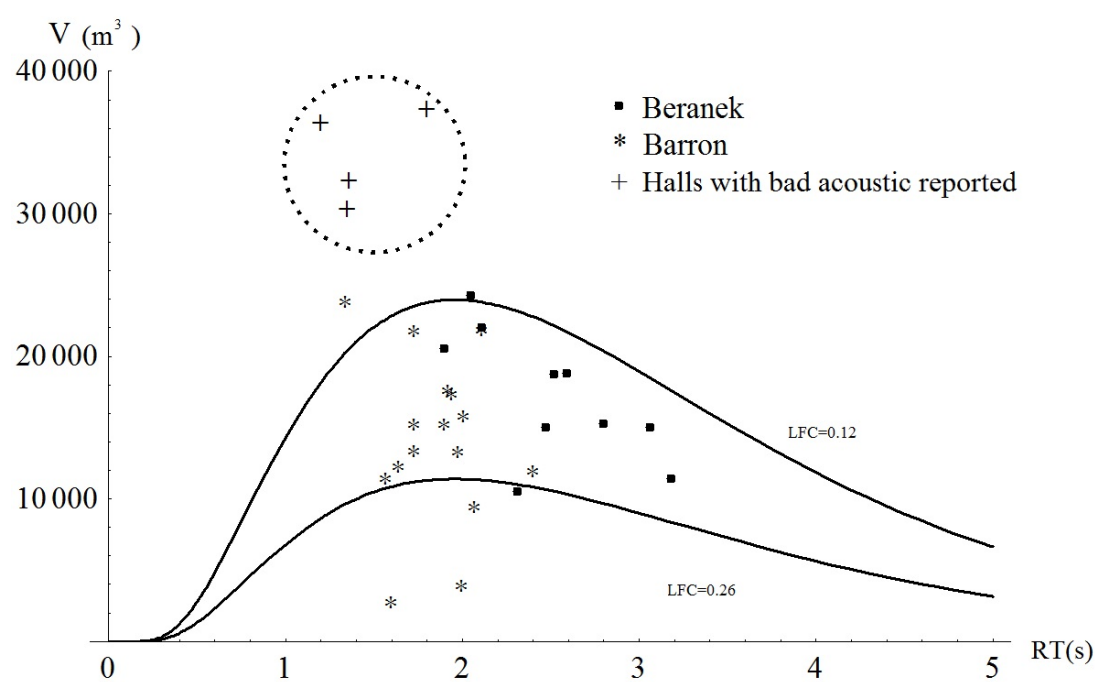

\section{Conclusions}

In this paper, we test Ando-Beranek's model of sound quality for symphony orchestra concert halls obtained by the authors in a previous work [17]. Ando-Beranek's model, a linear combination of T, LFC and LEV correlating with Ando functions of preferred values, was obtained after an extensive study of objective measures in room acoustics. First, an orthogonal group of parameters was statistical determined [13,14]. At the same time, a study of the subjective evaluation of a large number of rooms was conducted by surveying experts and concert-goers $[18,19]$. We gathered the results by statistical analysis and obtained the Ando-Beranek's model (5) with an optimal interval (6) for symphony orchestra concert halls.

Although traditional statistics is strong in devising ways of describing data and inferring distributional parameters from sample, the obtained statistical models can be spurious or have a poor validity. A way to show validity of the model is to apply it to another data set. A previous test of our model was presented in [21] where we used Ando-Beranek's model to evaluate quality in a hall by using quality maps.

In this paper, we have combined the Ando-Beranek's model with the Barron's theory. This has allowed us to obtain a formula that combines RT, LFC, IACCL3 and the Volume of the room. Using the minimum distance from the center of the scenario to the first line of seats as a reference, we have obtained expression (3). This expression can be represented (in Figure 2) showing that there is an optimal region in the V-RT plane for designing halls for classical music. This region has been successfully verified with well-known halls [7,20] and some halls with a regrettable reputation [24]. 


\section{Acknowledgments}

This work has been financially supported by FEDER funds and by the Ministry of Science and Technology with references Nos. BIA2003-09306, BIA2008-05485 and BIA 2012-36896.

\section{Author Contributions}

Authors are part of the Research Group in Virtual Acoustics (GIAV-UPV-UVEG) (http://www.upv.es/contenidos/ACUSVIRT/indexi.html), which is dedicated to study room acoustics, subjective assessment and virtualization of halls. This group is composed of people from the Universitat de València (UVEG) and the Universitat Politècnica de Valencia (UPV). The main reponsables of the group are Rosa Cibriàn in UVEG and Alicia Giménez at the UPV. Jaume Segura and Salvador Cerdá usually work on the analysis and processing of objective-subjective measurements and Arturo Barba is in charge of the study of the architectural features and geometric models.

\section{Conflicts of Interest}

The authors declare no conflicts of interest.

\section{References}

1. Bradley, J.S. Review of objective room acoustics measures and future needs. Appl. Acoust. 2011, 72, 713-720.

2. Sabine, W.C. Reverberation. Collected Papers on Acosutics; Hardvard University Press: Cambridge, MA, USA, 1922; pp. 3-68.

3. Lacatis, R.; Gimenez, A.; Barba Sevillano, A.; Cerda, S.; Romero, J.; Cibrian, R. Historical and chronological evolution of the concert hall acoustics parameters. J. Acoust. Soc. Am. 2008, 123, 3198-3204.

4. Ando, Y. Calculation of subjective preference at each seat in a concert hall. J. Acoust. Soc. Am. 1983, 74, 873-887.

5. Ando, Y. Concert Hall Acoustics; Springer: Berlin, Germany, 1985.

6. Ando, Y. Design Studies of Concert Halls as Public Spaces. In Auditory and Visual Sensations; Springer: Berlin, Germany, 2009; pp. 148-157.

7. Beranek, L. Concert Halls and Opera Houses: How They Sound? Woodbury: New York, NY, USA, 1996.

8. Kirkegaard, L.; Gulsrud, T. In search of a new paradigm: How do our parameters and measurement techniques constrain approaches to concert hall design? Acoust. Today 2011, 7, 7-14.

9. Lokki, T.; Patynen, J.; Kuusinen, A.; Vertanen, H.; Tervo, S. Concert hall acoustics assessment with individually elicited attributes. J. Acoust. Soc. Am. 2011, 130, 835-849.

10. Lokki, T.; Patynen, J.; Kuusinen, A.; Vertanen, H.; Tervo, S. Disentangling preference ratings of concert hall acoustics using subjective sensory profiles. J. Acoust. Soc. Am. 2012, 132, 3148-3161.

11. Lokki, T. Throw away that Standard and Listen: Your Two Ears Work Better. In Proceedings of International Symposium on Room Acoustics, Toronto, Canada, 9-11 June 2013. 
12. Cerda, S.; Cibrián, R.M.; Gimenez, A.; Girón, S.; Zamarreño, T. Mismatches between objective parameters and measured perception assessment in room acoustics: A holistic approach. Build. Environ. 2014, 74, 119-131.

13. Cerda, S.; Gimenez, A.; Romero, J.; Cibrian, R.M.; Miralles, J.L. Room acoustical parameters: A factor analysis approach. Appl. Acoust. 2009, 70, 97-109.

14. Cerda, S.; Gimenez, A.; Romero, J.; Cibrian, R.M. A factor analysis approach to determining a small number of parameters for characterising halls. Acta Acust. Unit. Acust. 2011, 97, 441-452.

15. Beranek, L.L. Concert Hall Acoustics-2008. J. Audio Eng. Soc. 2008, 56, 532-544.

16. Beranek, L.L. Listener Envelopment LEV, Strength G and Reverberation Time RT in Concert Halls. In Proceedings of 20th International Congress on Acoustics (ICA), Sydney, Australia, 23-27 August 2010; Volume 5, p. 4209.

17. Cerdá, S.; Giménez, A.; Cibrián, R. An objective scheme for ranking halls and obtaining criteria for improvements and design. J. Audio Eng. Soc. 2012, 60, 419-430.

18. Gimenez, A.; Cibrian, R.M.; Giron, S.; Zamarreño, T.; Sendra, J.J.; Vela, A.; Daumal, F. Questionnaire survey to qualify the acoustics of spanish concert halls. Acta Acust. United Acust. 2011, 97, 949-965.

19. Gimenez, A.; Cibrian, R.M.; Cerda, S. Subjective assessment of concert halls: A common vocabulary for music lovers and acousticians. Arch. Acoust. 2012, 37, 331-340.

20. Barron, M.; Lee, L.J. Energy relations in concert auditoriums I. J. Acoust. Soc. Am. 1988, 84, 618-628.

21. Cerda, S.; Segura, J.; Gimenez, A.; Cibrian, R.; Montell, R.; Barba, A. Sound Quality Maps of Halls for Classical Music. In Proceedings of the International Symposium on Room Acoustics, Toronto, Canada, 9-11 June 2013.

22. Cerda, S.; Segura, J.; Gimenez, A.; Cibrian, R. Concert rooms' quality for classical music. Inf. Constr. 2013, 65, 435-442 (in Spanish).

23. Beranek, L.L. Concert Halss and Opera Houses; Springer: New York, NY, USA, 2004.

24. O'Keefe, J. Acoustical problems in large post-war auditoria. Proc. Inst. Acoust. 2002, 24, Part 4.

25. Kutruff, H. Design Considerations and Design Procedures. In Room Acoustics; Spon Press: London, UK, 2009; pp. 294-328.

26. Siebein, G.W.; Kinzey, B.Y. Recent Innovations in Acoustical Design and Research. In Architectural Acoustics: Principles and Practice, 2nd ed.; Cavanaugh, W.J., Wilkes, J.A., Eds.; NCARB Monographs: Hoboken, NJ, USA, 2009.

27. Lokki, T. Tasting music like wine: Sensory evaluation of concert halls. Phys. Today 2014, 67, $27-32$.

28. O'Keefe, K. Influence of simple room geometries on acoustical parameters. J. Can. Acoust. Assoc. 1998, 26, 25-26.

29. Marshall, A.H. Levels of reflections masking in concert halls. J. Sound Vib. 1968, 7, 116-118.

(C) 2014 by the authors; licensee MDPI, Basel, Switzerland. This article is an open access article distributed under the terms and conditions of the Creative Commons Attribution license (http://creativecommons.org/licenses/by/3.0/). 